\title{
Relationships between antithyroperoxydase antibody levels, thyroid function and echographic patterns in chronic thyroiditis - December 2012
}

Mara Carşote ${ }^{1}$, Cătălina Poiană ${ }^{1}$, Cristina Daniela Staicu², Alexandrina Clodeanu², Dan Pereţianu $^{2}$

\section{Inst. Endocrinology "Parhon", 2. SCM "Povernei"; Bucharest - Romania}

Aim and objectives. To investigate the rapport between the level of thyroid function, through blood level of TSH, both at onset, and in evolution and the level of antithyroperoxydase antibodies (ATPO) and antithyroglobuline antibodies (ATG) in Hashimoto thyroiditis and related diseases.

Material\&Method. 1. Diagnosis: a. ATPO > $34 \mathrm{u} / \mathrm{ml}=$ Hashimoto thyroiditis (HT); b. ATPO=normal with high ATG = thyroiditis with only hyper-ATG (T-ATG); . hypothyroidism without high ATPO/ATG = idiopathic mixedema (IM). 2. Thyroid function: TSH (and FT4) 3. Echographic patterns $=\mathbf{1 0}$ (Peretianu, this Congress) .

Statistical analysis: linear correlation test, $\mathrm{X}^{2}$ test.

\section{Results.}

I. ATPO/ATG at onset: 1. ATPO: A. Number analysis: HT-1194, T-ATG-80 B. Average: TH-677,7; SD: 1190 (!); T-ATG-11,81; IM-10. 2. ATG in T-ATG: 483,23; SD: 852 (!).

II. Evolutional types for ATPO/ATG: 1. All ATPO in HT: no.2055, av: 740,17 ui/ml.

2. ATPO evolution in HT: undulatorious: 150 (40,31\%), decreasing: 156 (40,31\%), increasing: 81 $(20,93 \%)$. 3. All ATG in T-ATG: no. 100 , av: 415,71. 4. ATG in T-ATG: undulatorious: 4 (18,18\%), decreasing: 11 (50\%), increasing: $7(31,81 \%)$

III. TSH. 1. onset HT: av: $8,83 \mathrm{mu} / \mathrm{l} ; \quad 2$. onset T-ATG: $3,63 \mathrm{mu} / \mathrm{l}, \mathrm{SD}=4,33$.

3. Hypothyroidism HT: 41,12\%; Hypothyroidism T-ATG: $28,7 \%\left(\mathrm{X}^{2}=14,44, \mathrm{p}=0,044\right)$.

IV. Linear correlation ATPO-TSH: 1. in HT: a. HT at onset: $r=0,17, p<0,001$, slope: 8,31. b. all TH values: $r=0,11, p<, 001 ; \quad 2$. in T-ATG: a. at onset: $r=-0,19, p>0,1(\mathrm{NS})$; $b$. all T-ATG values: $r=-$ $0,17, \mathrm{NS} ; \mathrm{c}$. MI at onset/and all values, $\mathrm{r}=-0,17, \mathrm{p}>0,1$ (NS).

V. Echographic pattern 1 vs echographic pattern 8 in HT correlate with ATPO level (table): $\mathrm{X}^{2}>>$ 24,$9 ; p<<0,001$.

\begin{tabular}{|c|c|c|c|}
\hline $\begin{array}{l}\text { ATPO } \\
\text { levels }\end{array}$ & $\begin{array}{c}\text { Pattern 1 } \\
\text { marked hypoechogenous } \\
\text { pseudonodular }\end{array}$ & $\begin{array}{c}\text { Pattern } 8 \\
\text { only slightly hypoechogenous } \\
\text { pseudonodular }\end{array}$ & Total \\
\hline $0-34$ & 21 & 0 & 21 \\
\hline $34-100$ & 180 & 12 & 192 \\
\hline $100-350$ & 324 & 39 & 363 \\
\hline $350-999$ & 336 & 25 & 361 \\
\hline 1000-1999 & 244 & 10 & 254 \\
\hline 2000-4999 & 73 & 1 & 74 \\
\hline$>5000$ & 37 & 1 & 38 \\
\hline Total & 1215 & 88 & 1897 \\
\hline
\end{tabular}

1. A certain correlation exists between thyroid function but only for ATPO (not ATG), only in cohorts (not $<400$ probes), only in HT (not T-ATG): when ATPO increases, thyroid function decreases. 2 . HT vs T-ATG evolve with more hypothyroidism.

3. Pattern 8 is related low ATPO levels as compared with pattern 1. Therefore, pattern 1 suggest more inflammation than pattern 8 . 